\title{
Long-Acting, Injectable Antiretroviral Therapy for the Management of HIV Infection: An Update on a Potential Game-Changer
}

Linda Mobula ${ }^{1 *}$, Matt Barnhart ${ }^{1}$, Christine Malati ${ }^{1}$, Natella Rakhmanina ${ }^{2}$, Tom Minior ${ }^{1}$, Anouk Amzel $^{1}$, Robert Ferris ${ }^{1}$ and B Ryan Phelps ${ }^{1}$

'United States Agency for International Development, Washington, DC, USA

${ }^{2}$ Children's National Medical Center, Washington, DC, USA

\begin{abstract}
The efficacy of daily oral antiretroviral (ARVs), even when combined with supplemental adherence strategies, remains limited by non-adherence. Resistance and treatment failure resulting from missed ARV doses continue to hamper efforts to curb the epidemic. This commentary argues that we must simplify ARV regimens even further, and highlights the potential for long-acting injectable (LAl) formulations to facilitate weekly, monthly, and multi-month ARV regimens in both resource rich and resource poor settings. We summarize the latest progress in LAI development and reflect on the potential of LAls to revolutionize HIV management and define the next frontier of HIV medicine.
\end{abstract}

\section{Significant Advances in ARV Formulations are Still Limited by Poor Adherence}

Since the advent of zidovudine (AZT) to treat HIV infection over twenty-seven years ago, the number of available therapeutic choices has grown to include dozens of antiretroviral drugs (ARVs) spanning five therapeutic categories. [1]. The global scale-up of antiretroviral therapy is the combined achievement of scientists, clinicians, patients, advocates and pharmaceutical industry [2-4]. These ARVs not only improve life quality and expectancy, but also prevent perinatal and horizontal transmission of HIV infection. Alongside advances in development and approval of ARVs, the availability of these drugs in low- and middle- income countries continues to improve. Despite the progress that has been made in improving access to ARVs, adherence to the treatment drug regimens, like in other chronic disease, remains a complex challenge [5-7]. Behavioral constraints limiting long-term adherence to medications treating a variety of chronic diseases are well documented [8-13]. Worldwide, low rates of adherence to ARV regimens combined with treatment interruptions continue to limit the effectiveness of HIV programs in reaching epidemic control. The combination of non-adherence and treatment interruptions leads to suboptimal community viral suppression, increased HIV spread, higher rates of morbidity and mortality and HIV drug resistance. Such resistance in turn requires increased use of more expensive second line drugs [14,15]. Similarly, the efficacy and safety of pre-exposure prophylaxis, a key tool to reduce the incidence of HIV infection in high-risk individuals, has been shown to be compromised by inconsistent dosing, leading to increases HIV transmission and resistance in the presence of sub-therapeutic drug levels $[11,12,15]$.

\section{The Promise of Drug Simplification}

Due to these factors, non-adherence to ART remains among the most formidable obstacles to an AIDS-free generation. To minimize the liability that non-adherence represents and to lower the burden on patients on treatment, there remains a focus on simpler drugs that can be taken less often $[16,17]$. Simplified strategies have been demonstrated to improve adherence and patient satisfaction in other chronic disease management schema, including cardiovascular disease, hypertension, elevated cholesterol, thrombosis, depression, and osteoporosis [18-33]. In recent decades, increasingly simplified contraception methods have brought with them higher efficacy and patient convenience [34].

\section{Simplifying ARVs}

In the current context of HIV, the recommended first-line ART can be prescribed as a once-daily single fixed dose combination (FDC) tablet, representing a significant improvement in the pill burden and cost compared to previously available regimens (Table 1). Consistent with adherence studies across other chronic diseases, studies in the context of HIV suggest significant benefits to adherence and quality of life with simplified, once daily regimens as compared with twice daily, multi-pill regimens [35].

An effort to further widen the allowable time between ARV doses has led to the development of extended release oral formulations. The only currently available drug in this category is nevirapine XR, which was approved by the FDA in March 2011. The so-called VER $\times$ VE and TRAN $\times$ TION clinical trials established the clinical non-inferiority of NVP XR in both treatment-naive and treatment-experienced patients [36,37]. Since approval, extended release nevirapine has been demonstrated to improve adherence, patient satisfaction and reduce the risk of resistance and virologic failure. Additionally, it has been shown to increase efficacy and decrease the incidence and intensity of adverse effects [38,39].

However, despite ARV simplification and a diversity of related strategies to reinforce day-to-day ARV adherence behaviors (directly

\begin{tabular}{|l|l|l|}
\hline & $\mathbf{2 0 0 2}$ & $\mathbf{2 0 1 3}$ \\
\hline First-Line Regimen for Adults & $\begin{array}{l}\text { AZT/3TC with } \\
\text { NVP or EFV }\end{array}$ & $\begin{array}{l}\text { TDF/FTC/EFV or } \\
\text { TDF/3TC/EFV }\end{array}$ \\
\hline Minimum Daily Pill Burden & 3 tablets & 1 tablet \\
\hline $\begin{array}{l}\text { Monthly Price for Minimum Pill Burden } \\
\text { Regimen }\end{array}$ & $\$ 32.28$ & $\$ 11.38$ \\
\hline
\end{tabular}

Table 1: Differences Between the Price and Daily Pill Burden Between the First Line Regimen in 2002 and that from 2013.

*Corresponding author: Linda Mobula, Office of HIVIAIDS, United States Agency for International Development, Washington, DC, USA, Tel: 571-551-7303; E-mail: mmobula@usaid.gov

Received April 30, 2015; Accepted May 25, 2015; Published June 04, 2015

Citation: Mobula L, Barnhart M, Malati C, Rakhmanina N, Minior T, et al. (2015) LongActing, Injectable Antiretroviral Therapy for the Management of HIV Infection: An Update on a Potential Game-Changer. J AIDS Clin Res 6: 466. doi:10.4172/21556113.1000466

Copyright: $\odot 2015$ Mobula L, et al. This is an open-access article distributed under the terms of the Creative Commons Attribution License, which permits unrestricted use, distribution, and reproduction in any medium, provided the original author and source are credited. 
observed therapy, treatment buddies, support groups, electronic reminder systems, etc.), adherence rates to daily regimens are inherently limited by predictable human behavior [40,41]. To boost lasting adherence, it is necessary to recognize this stubborn fact, and further simplify ARV regimens. The next logical step toward simpler, easier HIV therapy is the development of effective, affordable longer acting ARVs. In the absence of an HIV cure, we believe such ARVs, in the form of long-acting injectable (LAI) formulations, will define the future of life-long HIV management.

\section{Long-Acting Injectibles (LAIs)}

While the precedent for long-acting injectables in chronic disease management is limited, several key successes stand out. Use of long acting injectable antipsychotics for the treatment of schizophrenia have led to decreased hospitalization rates, improved adherence, treatment outcomes, patient satisfaction and better quality of life [21, 24,30,4144]. The use of long-acting injectable glucagon-like peptide- 1 receptor (GLP-1R) agonists for the treatment of diabetes has been shown to improve glycemic control, glycolated hemoglobin, promote weight loss as well as reduce cardiovascular risk by improving blood pressure control and lipid levels [45-47]. Injectable hormones have become the mainstay of long-acting contraception, decreasing unintended pregnancies and abortions while achieving lower failure rates in both resourced and under resourced countries [34,49,50].

\section{Advantages of LAIs}

The critical advantage to LAIs, of course, is that they could be dosed on a weekly, monthly, or multi-month schedule. Compared to oral dosing, LAIs would likely have reduced drug interactions related to first-pass metabolism, as well as decreased gastrointestinal toxicity [50]. Also, injectable formulations with specific target cells such as $\mathrm{CD}_{4}$ cells would require lower doses, potentially reducing costs of ARVs in resource limited settings where the active pharmaceutical ingredient compound represents a large proportion of the total production costs [50,51]. The availability of long acting parental ART formulations would benefit critically ill, hospitalized and perioperative patients who are unable to tolerate oral medications. Among populations that are particularly at risk for ARV non-adherence, LAIs would provide another option for healthcare providers who might be reluctant to prescribe oral ARVs due to anticipated adherence concerns [52]. Though previous studies have demonstrated that once-daily FDC is associated with improved adherence, it will be important to conduct behavioral studies to demonstrate this.

\section{Predictable Challenges/Disadvantages of LAIs}

As stated above, LAI formulations typically have a longer time (several days rather than hours) to maximum concentration $\left(\mathrm{T}_{\max }\right)$ when compared of oral formulations, followed by sustained plasma concentration near peak concentrations $\left(\mathrm{C}_{\max }\right)$ [53-55]. For this reason, then starting a LAI, a period of oral induction therapy is often required help ensure achievement of appropriate drug concentrations while the LAI approaches $\mathrm{T}_{\max }$. Oral induction also helps scout for hypersensitivity or drug toxicity, so that long-lasting adverse events might be avoided. Also, the prolonged clearance time of LAIs increase the risk of prolonged low drug concentrations in the context of missed doses. In sum, LAIs are likely to decrease adherence burden, but also raise the stakes in regards to adverse events and non-adherence. Please refer to Table 2 which describes additional challenges.

\section{A Proof of Concept: Promising ARV LAIs in Clinical Development}

Currently, two LAI ARV formulations - long-acting (LA) versions of a second generation non-nucleoside reverse transcriptase inhibitor (NNRTI), rilpivirine, and an integrase inhibitor, cabotegravir (formerly GSK744) - are in clinical-stage development. Both formulations are intended to be given monthly (or less frequently) and have been prepared using nanomilling technology. This nanomilling technology approach has already been already successfully applied to several FDA approved pharmaceutical formulations for other diseases, including: abraxane for the treatment of breast, pancreatic and small lung cell lung cancer; and doxil for the treatment of multiple myeloma and ovarian cancer [53-55].

Additionally, researchers are laying the ground work for assessment of combination LA ARVs for use as a maintenance regimen. Phase $2 b$ of the Long-Acting Antiretroviral Treatment Enabling (LATTE) trial is currently evaluating oral cabotegravir plus rilpivirine as a 2-drug maintenance regimen among patients who have already achieved

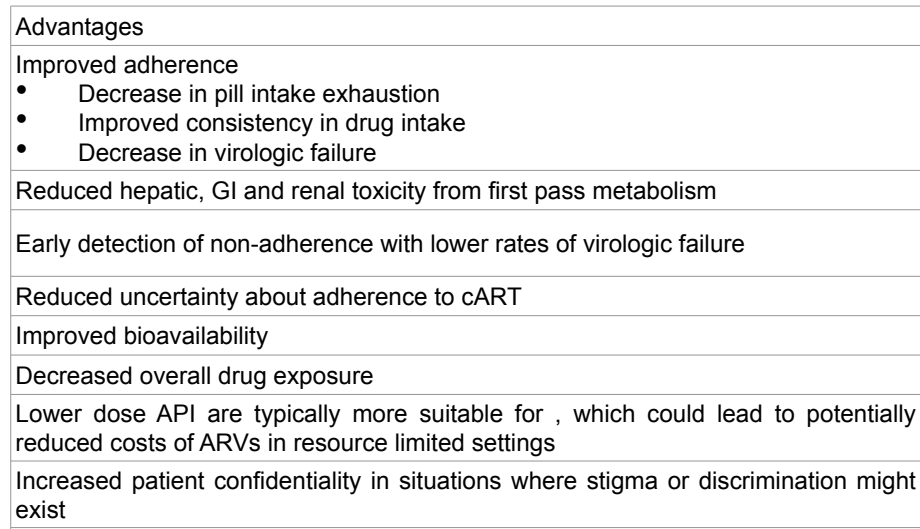

Can be considered for PreP use

Nanoemulsions can be injected with reduced volume

Possibility for painful subcutaneous or subdermal implantation
Disadvantages

Pain, erythema, nodule formation at site injection

Need for competent injection technique and for trained health care workers

Long periods of low drug concentration if injection is missed, likely leading to high risk of resistance

Longer time to maximum concentration (can be dealt with oral lead in period)

Potential for development of resistance when doses are missed

Difficulty with withdrawal of drug in the event of toxicity

Need for increased clinic visits with monthly injections versus quarterly visits for stable ART patients on oral medications

Improved cost with lesser need for active ingredient

Use as a PreP option could potentially lead to resistance given subtherapeutic levels if HIV is acquired

Manufacturing of antibodies can be costly

Missing a dose may lead to longer coverage-free periods compared to oral doses

Much shorter tail of drug exposure in cases where loss-to-follow-up occurs 
viral suppression [56]. The LATTE trial has demonstrated that both drugs, when administered orally, were as effective and better tolerated in comparison to triple ART regimen containing efavirenz plus two NRTIs. Once these studies looking at the effectiveness and tolerability of the oral two-drug combination are complete, the researchers aim in subsequent studies to do a similar assessment with the LAI versions of the drugs [57].

When looking at the LAI plasma drug concentrations, a study conducted by Spreen et al. demonstrated that plasma drug concentrations achieved with the oral formulations were also achievable with an LAI formulation [58]. Following respective loading doses, cabotegravir consistently maintained high plasma concentrations (4-fold above the protein-adjusted inhibitory concentration (IC)-90 and comparable to steady-state oral RPV $25 \mathrm{mg} / \mathrm{d}$ ) associated with monthly injections or quarterly intramuscular injections. For over 30 days, a $600-900 \mathrm{mg}$ dose of rilpivirine LAI maintained plasma concentrations above the plasma trough levels observed with $25 \mathrm{mg}$ daily oral rilpivirine.

Rilpivirine and cabotegravir represent a promising LAI combination for several reasons. Firstly, they appear to have good tolerability profiles; co-administration of these two ARV Nano suspensions was found to be safe and well tolerated in healthy human adults [59] Secondly, cabotegravir has significantly higher resistance barrier than rilpiverine, and resulting resistance mutations confer low-level resistance without cross resistance to other integrate inhibitors. A tail containing cabotegravir LAI therefore offers some protection against the emergence of high-level resistance. Thirdly, the cost of producing this rilpivirine/cabotegravir combination is likely to be relatively affordable. Based on previous manufacturing estimates, rilpvirine LA and a theoretical formulation of dolutegravir LA (an integrase inhibitor similar to cabotegravir) would cost approximately $\$ 40$ per year [35].

\section{Proceeding With Caution: Moving Rilpivirine and Cabotegravir Toward Regulatory Approval}

Though a combination LAI containing rilpivirine and cabotegravir provides the first proof-of-concept that LAIs can become a safe and effective alternative to oral regimens, this is just the beginning. The tolerability of this first generation combination LAI is limited by a relatively high (1-4 ml) injection volume, which can be painful when administered intramuscularly. The rilpivirine/cabotegravir formulation has a very long tail of drug exposure in the body (lasting up to a year) and therefore brings with it a high likelihood that resistance will develop in those lost to follow-up. As such an example, one case of rilpivirine-induced NNRTI resistance has already been reported in an initially HIV-uninfected trial participant who received rilpivirine LA for pre-exposure prophylaxis. The individual was then found to be infected months later, during the tail period [60]. Finally, while the manufacture costs of these two LAIs ARVs might be less than cost of currently recommended oral first line, other costs may increase the true costs the Nano milling process, injection equipment, and costs to the health system (training, personnel, gloves, etc.).

\section{Conclusion}

\section{Looking ahead at the future of ARVs}

Daily HIV treatment regimens remain an irrefutable and arguably insurmountable barrier to adherence and, ultimately, viral suppression and epidemic control. We must therefore identify strategies to ensure that we adequately target these barriers. As a rilpivirine/cabotegravir LAI moves toward regulatory approval, it is important to ask several critical questions.

\section{1) How do we nurture further LAI development?}

If ARV LAI regimens are to be truly transformative, their development needs to be prioritized. Significant improvements beyond first-generation ARV LAI combinations will be needed, and LAI development should be incentivized, including support of LAI costeffectiveness studies.

\section{2) Are there other ARVs that hold promise for inclusion in LAIs?}

Prioritization of appropriate drugs for LAI development will require thoughtful planning. Stakeholders should invest in a longterm, concerted effort to carefully select promising pharmaceutical ingredients and design optimal drug delivery systems. For example, cabotegravir's many promising characteristics may lead to the selection of a compatible, efficacious partner drug for future LAI combinations. This partner drug would need to possess high potency and be concentrated in a much lower injection volume. It would need a high resistance barrier and tolerability along with a limited number of interactions with medications commonly utilized in resource-limited settings, including antimalarial, TB drugs and opportunistic infection prophylaxis.

\section{3) How and where might we pilot ARV LAI implementation?}

Assessments of LAIs are needed in both resource limited and resourced settings. Implementation of the first generation of LAIs in resource limited settings, even on a relatively small scale, would provide important lessons and inform good practices and approaches for improving development of subsequent generations of LAIs. Now is the time to consider how to design this pilot, with a focus on populations that typically experience high rates of poor adherence, ideally in highburden, lower resourced settings. A pilot is also needed in resourced settings, perhaps targeting populations that are known for adherence issues and that would most benefit from LAIs, such as injection drug users [52].

\section{4) Beyond LAIs, what long-acting approaches show promise?}

LAI nanoemulsions, though poised to play a critical role in the further simplification of HIV treatment, are best suited to deliver hydrophobic compounds [62,63]. An alternative to injection is a transcutaneous delivery using a thin film biodegradeable device made with the FDA-approved polymer called poly caprolactone, which can hold solid formulations of active pharmaceutical ingredients, and can be applied directly to skin [63]. Potential advantages of this approach include reduced drug volume, avoidance of painful injection, potential for longer-term activity, and a shorter tail of drug exposure in cases where loss-to-follow-up occurs.

\section{5) What about broadly neutralizing antibodies?}

Finally, broadly neutralizing antibodies (bnAbs) against HIV represent another promising area. Custom-engineered bnAbs hold immense promise and, high manufacturing costs, remain an active area of research.

While it is clear that LAIs and other long-acting treatment regimens will not become the mainstay of HIV treatment for years to come, they certainly have the potential to someday revolutionize HIV management. In the absence of an HIV cure, and with appropriate planning and support, LAIs are well-positioned to redefine HIV treatment regimens, define the next frontier of HIV medicine, and save and prolong the lives of millions. How quickly they advance from concept to game-changer is up to us. 
Citation: Mobula L, Barnhart M, Malati C, Rakhmanina N, Minior T, et al. (2015) Long-Acting, Injectable Antiretroviral Therapy for the Management of HIV Infection: An Update on a Potential Game-Changer. J AIDS Clin Res 6: 466. doi:10.4172/2155-6113.1000466

\section{References}

1. http://www.fda.gov/forpatients/illness/hivaids/treatment/ucm895.htm.

2. Pipeline Report (2003) HIV, HCV, and TB Drugs, Diagnostics, Vaccines, and Preventive Technologies in Development. Treatment Action Group.

3. Vitoria M, Vella S, Ford N (203) Scaling up antiretroviral therapy in resourcelimited settings: adapting guidance to meet the challenges. Curr Opin HIV AIDS 8: $2-8$.

4. Global HIVIAIDS Response (20) Epidemic Update and Health Sector Progress towards Universal Access Progress Report. UNAIDS

5. Kauf TL, Davis KL, Earnshaw SR, Davis EA (202) Spillover adherence effects of fixed-dose combination HIV therapy. Patient Prefer Adherence 6: 55-64

6. Merten S, Kenter E, McKenzie O, Musheke M, Ntalasha H, et al. (200) Patientreported barriers and drivers of adherence to antiretrovirals in sub-Saharan Africa: a meta-ethnography. Trop Med Int Health 5 Suppl : 6-33.

7. Reginster JY, Rabenda V, Neuprez A (2006) Adherence, patient preference and dosing frequency: understanding the relationship. Bone 38: S2-6.

8. Claxton AJ, Cramer J, Pierce C (200) A systematic review of the associations between dose regimens and medication compliance. Clin Ther 23: 296-30.

9. Mills EJ, Nachega JB, Buchan I, Orbinski J, Attaran A, et al. (2006) Adherence to antiretroviral therapy in sub-Saharan Africa and North America: a metaanalysis. JAMA 296: 679-690.

10. Fox M , Rosen S (200) Patient retention in antiretroviral therapy programs up to three years on treatment in sub- Saharan Africa, 2007-2009: a systematic review. Tropical Medicine and International Health 5: -5

11. Koenig LJ, Lyles C, Smith DK (203) Adherence to antiretroviral medications for HIV pre-exposure prophylaxis: lessons learned from trials and treatment studies. Am J Prev Med 44: S9-98.

12. Ware NC, Wyatt MA, Haberer JE, Baeten JM, Kintu A, et al. (202) What's love got to do with it? Explaining adherence to oral antiretroviral pre-exposure prophylaxis for HIV-serodiscordant couples. J Acquir Immune Defic Syndr 59: 463-468.

13. Gupta R, Jordan M, Binta JS, Andrew H, Daniel H, et al. (202) Global trends in antiretroviral resistance in treatment-naïve individuals with HIV after rollout of antiretroviral treatment in resource-limited settings: a global collaborative study and meta-regression analysis. Lancet 330: 250-258

14. Hosseinipour MC, Gupta RK, Van Zyl G, Eron JJ, Nachega JB (203) Emergence of HIV drug resistance during first- and second-line antiretroviral therapy in resource-limited settings. J Infect Dis 207 Suppl 2: S49-56.

15. Mutua G, Sanders E, Mugo P, Anzala O, Haberer JE, et al. (202) Safety and adherence to intermittent pre-exposure prophylaxis (PrEP) for HIV- in African men who have sex with men and female sex workers. PLoS One 7: e3303.

16. Airoldi M, Zaccarelli M, Bisi L, Bini T, Antinori A, et al. (200) One-pill once-a-day HAART: a simplification strategy that improves adherence and quality of life of HIV-infected subjects. Patient Prefer Adherence 4: 5-25.

17. Bangsberg DR, Ragland K, Monk A, Deeks SG (200) A single tablet regimen is associated with higher adherence and viral suppression than multiple tablet regimens in $\mathrm{HIV}+$ homeless and marginally housed people. AIDS 24: 28352840 .

18. Altice FL, Kamarulzaman A, Soriano VV, Schechter M, Friedland GH (200) Treatment of medical, psychiatric, and substance-use comorbidities in people infected with HIV who use drugs. Lancet 376: 367-387.

19. Lemstra M, Blackburn D, Crawley A, Fung R (202) Proportion and risk indicators of nonadherence to statin therapy: a meta-analysis. Can J Cardiol 28: 574-580.

20. Brnabic AJ, Kelin K, Ascher-Svanum H, Montgomery W, Kadziola Z, et al. (2011) Medication discontinuation with depot and oral antipsychotics in outpatients with schizophrenia: comparison of matched cohorts from a 2-month observational study. Int J Clin Pract 65: 945-953.

21. de Klerk E (2001) Patient compliance with enteric-coated weekly fluoxetine during continuation treatment of major depressive disorder. J Clin Psychiatry 62 Suppl 22: 43-47.

22. Marinis DT, Saleem PT, Glue P, Arnoldussen WJ, Teijeiro R, et al. (2007) Switching to long-acting injectable risperidone is beneficial with regard to clinical outcomes, regardless of previous conventional medication in patients with schizophrenia. Pharmacopsychiatry 40: 257-263
23. Kaplan G, Julio C, Jacqueline Z (2013) Impact of long-acting injectable antipsychotics on medication adherence and clinical, functional, and economic outcomes of schizophrenia. Patient Prefer Adherence 7: 7-80

24. Grabner M, Chu J, Raparla S, Quimbo R, Zhou S, et al. (2013) Clinical and economic outcomes among patients with diabetes mellitus initiating insulin glargine pen versus vial. Postgrad Med 25: 204-23

25. Barner JC (2011) Adherence to oral antidiabetic agents with pioglitazone and metformin: comparison of fixed-dose combination therapy with monotherapy and loose-dose combination therapy. Clin Ther 33: 28-288.

26. Lemstra M, Blackburn D, Crawley A, Fung R (2012) Proportion and risk indicators of nonadherence to statin therapy: a meta-analysis. Can $\mathrm{J}$ Cardio 28: $574-580$.

27. Brnabic AJ, Kelin K, Ascher-Svanum H, Montgomery W, Kadziola Z, et al. (2011) Medication discontinuation with depot and oral antipsychotics in outpatients with schizophrenia: comparison of matched cohorts from a 2-month observational study. Int J Clin Pract 65: 945-953.

28. de Klerk E (2001) Patient compliance with enteric-coated weekly fluoxetine during continuation treatment of major depressive disorder. J Clin Psychiatry 62: 43-47.

29. Thom S, Poulter N, Field J, Patel A, Prabhakaran D, et al. (2013) Effects of a fixed-dose combination strategy on adherence and risk factors in patients with or at high risk of CVD: the UMPIRE randomized clinical trial. JAMA 30: 98-929.

30. Westhoff C, Heartwell S, Edwards S, Zieman M, Cushman L, et al. (2007) Initiation of oral contraceptives using a quick start compared with a conventional start: a randomized controlled trial. Obstet Gynecol 09: 270-276.

31. Flexner C, Tierney C, Gross R, Andrade A, Lalama C, et al. (2010) Comparison of Once-daily versus twice-daily combination antiretroviral therapy in treatmentnaïve patients: results of AIDS Clinical Trials Group A5073, a 48-week randomized control trial. Clinical Infectious Diseases 50: 1041-1052.

32. Gathe J, Andrade-Villanueva J, Santiago S, Horban A, Nelson M, et al. (2011) Efficacy and safety of nevirapine extended-release once daily versus nevirapine immediate-release twice-daily in treatment-naive HIV--infected patients. Antivir Ther 6: 759-769.

33. Arasteh K, Ward D, Plettenberg A, Livrozet JM, Orkin C, et al. (2012) Twentyfour-week efficacy and safety of switching virologically suppressed HIVinfected patients from nevirapine immediate release $200 \mathrm{mg}$ twice daily to nevirapine extended release $400 \mathrm{mg}$ once daily (TRANxITION) HIV Med 13 236-244

34. Mateo MG, Gutierrez Mdel M, Vidal F, Domingo P (2014) Stavudine extended release (once-daily, Bristol-Myers Squibb) for the treatment of HIVIAIDS. Expert Opin Pharmacother 14:1055-1064.

35. Laufs U, Böhm M, Kroemer HK, Schüssel K, Griese N, et al. (2011) [Strategies to improve medication adherence]. Dtsch Med Wochenschr 36: 66-62.

36. Libby AM, Fish DN, Hosokawa PW, Linnebur SA, Metz KR, et al. (2013) Patient-level medication regimen complexity across populations with chronic disease. Clin Ther 35: 385-398.

37. Offord S, Wong B, Mirski D, Baker RA, Lin J (2013) Healthcare resource usage of schizophrenia patients initiating long-acting injectable antipsychotics vs oral. J Med Econ 6: 23-239.

38. Witte MM, Case MG, Schuh KJ, Ascher-Svanum H (2012) Effects of olanzapine long-acting injection on levels of functioning among acutely ill patients with schizophrenia. Curr Med Res Opin 28: 35-323.

39. Lloyd K, Latif MA, Simpson S, Shrestha KL (2001) Switching stable patients with schizophrenia from depot and oral antipsychotics to long-acting injectable risperidone: efficacy, quality of life and functional outcome. Hum Psychopharmacol 25: 243-252.

40. Verdoux H, Lengronne J, Liraud F, Gonzales B, Assens F, et al. (2000) Medication adherence in psychosis: predictors and impact on outcome. 2-year follow-up of first-admitted subjects. Acta Psychiatr Scand 02: 203-20.

41. Drucker DJ, Buse JB, Taylor K, Kendall DM, Trautmann M, et al. (2008) Exenatide once weekly versus twice daily for the treatment of type 2 diabetes: a randomised, open-label, non-inferiority study. Lancet 372: 240-250.

42. Wysham C, Grimm M, Chen S (2013) Once weekly exenatide: efficacy, tolerability and place in therapy. Diabetes Obes Metab 5: 87-88. 
Citation: Mobula L, Barnhart M, Malati C, Rakhmanina N, Minior T, et al. (2015) Long-Acting, Injectable Antiretroviral Therapy for the Management of HIV Infection: An Update on a Potential Game-Changer. J AIDS Clin Res 6: 466. doi:10.4172/2155-6113.1000466

Page 5 of 5

43. Meloni AR, DeYoung MB, Han J, Best JH, Grimm M (2013) Treatment of patients with type 2 diabetes with exenatide once weekly versus oral glucose-lowering medications or insulin glargine: achievement of glycemic and cardiovascular goals. Cardiovasc Diabetol 2: 48.

44. Secura G (2013) Long-acting reversible contraception: a practical solution to reduce unintended pregnancy. Minerva Ginecol 65: 27-277.

45. Prescott GM, Matthews CM (2014) Long-acting reversible contraception: a review in special populations. Pharmacotherapy 34: 46-59.

46. Boffito M, Jackson A, Owen A, Becker S (2014) New approaches to antiretroviral drug delivery: challenges and opportunities associated with the use of longacting injectable agents. Drugs 74: 7-3.

47. Siccardi M, Martin P, McDonald TO, Liptrott NJ, Giardiello M, et al.(2013) Nanomedicines for HIV therapy. Future Science 4: 153-156

48. Williams J, Sayles HR, Meza JL, Sayre P, Sandkovsky U et al. (2013) Longacting parenteral nanoformulated antiretroviral therapy: interest and attitudes of HIV-infected patients. Nanomedicine (Lond)8: 1807-1813

49. http://www.fda.gov/ForConsumers/ByAudience/ForPatientAdvocates/ HIVandAIDSActivities/ucm895.htm

50. http://www.fda.gov/Drugs/InformationOnDrugs/ApprovedDrugs/ucm323668 htm.

51. http://www.fda.gov/newsevents/newsroom/pressannouncements/ucm367442. htm

52. Margolis D, Cynthia B, Joseph E, Gary R, Roger L, et al. (2014) 744 and Rilpivirine as Two-Drug Oral Maintenance Therapy: LAI6482 (LATTE) Week 48 Results. 2st Conference on Retroviruses and Opportunistic Infections ,Boston

53. Margolis DA, Sandy G, Marty SC, William S, Cynthia B, et al. (2015) Cabotegravir and Rilpivirine as 2-Drug Oral Maintenance Therapy: LATTE W96 Results. 205 Conference on Retroviruses and Opportunistic Infections, Seattle

54. Spreen W, Williams P, Margolis D, Ford SL, Crauwels H, et al. (204)
Pharmacokinetics, safety, and tolerability with repeat doses of GSK265744 and rilpivirine (TMC278) long-acting nanosuspensions in healthy adults. J Acquir Immune Defic Syndr 67: 487-492.

55. Spreen W, Williams P, Margolis D, Ford S, Crewels H, et al. (2013) Firs study of repeat dose co administration of GSK265744 and TMC278 long acting parenteral Nano suspensions: pharmacokinetics, safety and tolerability in healthy adults. 7th IAS Conference on HIV Pathogenesis, Treatment and Prevention, Kuala Lumpur.

56. Penrose KJ, Parikh UM, Hamanishi KA, Panousis C, Else L, et al. (2014) Selection of Rilpivirine Resistant HIV- in a Seroconverter on Long-acting Rilpivirine (TMC278LA) from the Lowest Dose Arm of the SSAT 040 Trial. AIDS research and human retroviruses 30: A69.

57. Liptrott N, Philip Martin,McDonald, Giardiell M, Roberts P, et al. (2013) Enhanced pharmacological properties of efavirenz formulated as solid drug nanoparticles. 20th CROI, Atlanta.

58. Tapan K. Dash, Konkimalla VB (2012) “Poly-€-caprolactone based formulations for drug delivery and tissue engineering: A review. Journal of Controlled Release 158: 15-33

59. Sources and Prices of Selected Medicines and Diagnostics for People Living with HIVIAIDS: A Joint UNICEF-UNAIDS-WHO-MSF Project.

60. Piacenti FJ (2006) An update and review of antiretroviral therapy. Pharmacotherapy 26: 1111-1133.

61. Bendavid E, Leroux E, Bhattacharya J, Smith N, Miller G (200) The relation of price of antiretroviral drugs and foreign assistance with coverage of HIV treatment in Africa: retrospective study. BMJ 34: c628.

62. WHO (2013) Antiretroviral Therapy for HIV Infection in Adults and Adolescents: Recommendations for a Public Health Approach.

63. Spreen WR, Margolis DA, Pottage JC Jr (203) Long-acting injectable antiretrovirals for HIV treatment and prevention. Curr Opin HIV AIDS 8: 565-57. 